\title{
High Speed Keck Spectroscopy of Flickering in AM Her
}

\author{
Warren Skidmore \\ Physics and Astronomy, University of California Irvine, 4129 Frederick \\ Reines Hall, Irvine, CA 92697-4575, USA. \\ Richard Gomer \\ Howard Hughes Medical Institute, Department of Biochemistry and Cell \\ Biology, MS-140, Rice University, Houston, TX 77005-1892, USA. \\ Keith Horne \\ School of Physics and Astronomy, University of St. Andrews, North \\ Haugh, St. Andrews, Fife, KY16 9SS, UK. \\ Kieran O'Brien \\ E.S.O., Alonso de Cordova 3107, Casilla 19001, Santiago 19, Chile. \\ B. Oke
}

Dominion Astrophysical Obs., 5071 West Saanich Road, Victoria, BC, Canada

\author{
K.J. Pearson \\ Louisiana State University, Department of Physics and Astronomy, \\ Nicholson Hall, Baton Rouge, LA 70803-4001, USA
}

\begin{abstract}
We present fast (72ms) spectroscopy of AM Her obtained at an intermediate brightness state just before a rise to a high state. Interesting features in the line behaviour of AM Her are noted and the variability spectrum is presented and compared to that of SS Cyg.
\end{abstract}

\section{Introduction}

Doppler tomograms of most polars show a narrow emission line component arising from the secondary star as well as line emission from the ballistic and magnetically channeled parts of the accretion stream (Schwope 2001). However Schwarz et al. (2002) found that in AM Her, the prototype polar system, the emission line flux from material in the accretion stream does not follow the simple ballistic trajectory even at the $L_{1}$ point.

Beardmore \& Osborne (1997) suggested that the accretion onto the magnetic pole of AM Her consists of discrete blobs of material. In order for this to happen, blobs must remain coherent as they travel along the accretion stream. The travel time for these blobs is approximately 10 minutes. During that time 


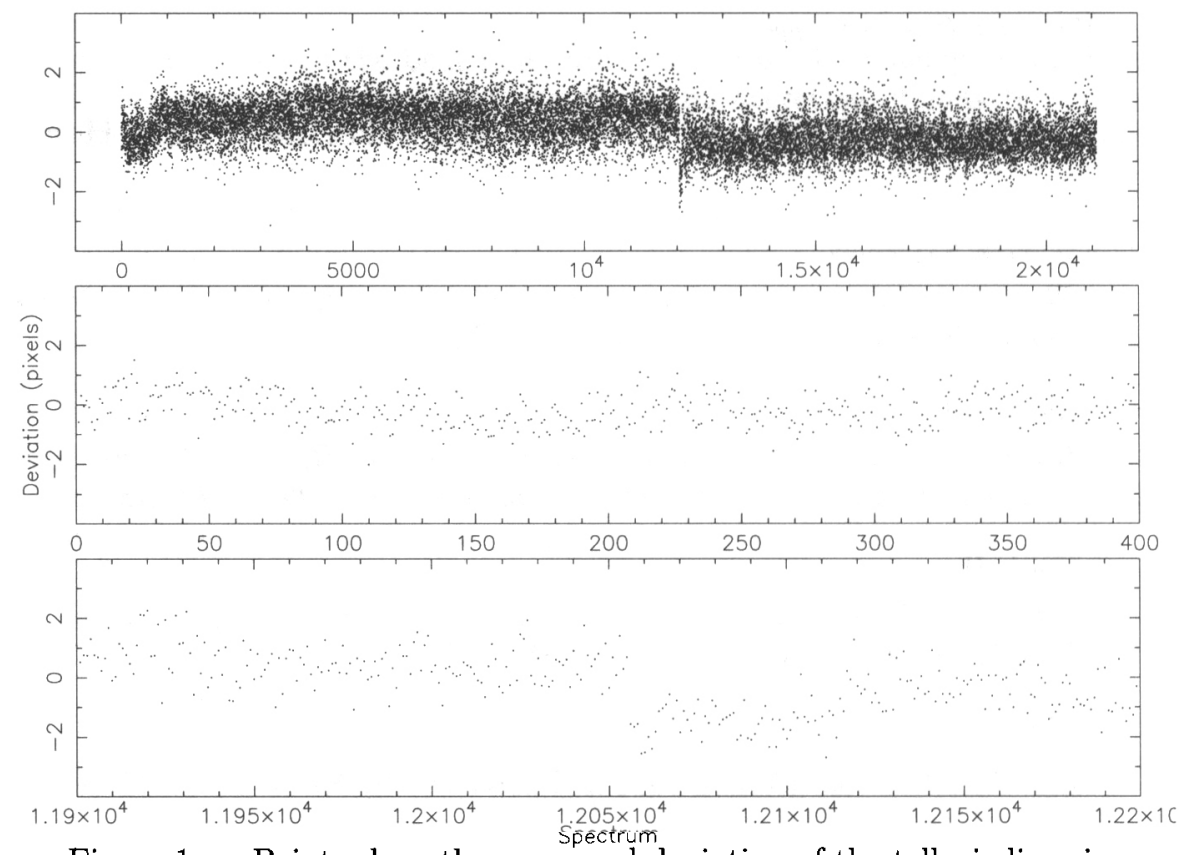

Figure 1. Points show the measured deviation of the telluric lines in each spectrum w.r.t. the mean spectrum. Top panel: complete data set. Middle and bottom panels: close up to show short-term trends and large scale jumps.

many processes occur, such as capture by the field lines and the final impact onto the pole. Doppler tomography of AM Her (Schwarz 2002) could not resolve the signature of individual blobs. It is a search for observational signatures of these blobs that motivated the observations of AM Her described here.

In polars, the gas flow from the secondary initially follows the ballistic trajectory until the stream encounters the white dwarf magnetosphere. The gas velocity then changes as the flow is captured by the field and forced to flow along the field lines to the white dwarf surface. X-rays emitted at the white dwarf surface illuminate the incoming stream and cause the strong emission lines seen in the optical spectrum (see Figure 3). In current models, the optical emission lines arise from reprocessing of X-rays on the companion star and accretion stream. The optical flares and flickers represent changes in X-ray illumination, or in the geometry of the accretion stream.

\subsection{The Data Acquisition System and Data Analysis}

The LRIS on the Keck II Telescope was used in a novel manner to obtain very fast readout times ( $72 \mathrm{~ms}$ per spectrum) of medium resolution spectra $(2.4 \AA$ per pixel) during a campaign to observe LMXBs and CVs in 1998. AM Her was observed for 28 minutes, yielding over 21000 spectra.

The CCD was read out in a continuous drift mode with each row corresponding to a single $72 \mathrm{~ms}$ spectrum. Using a spectrograph in this manner introduced problems not seen with normal spectrographs. The size of the spec- 

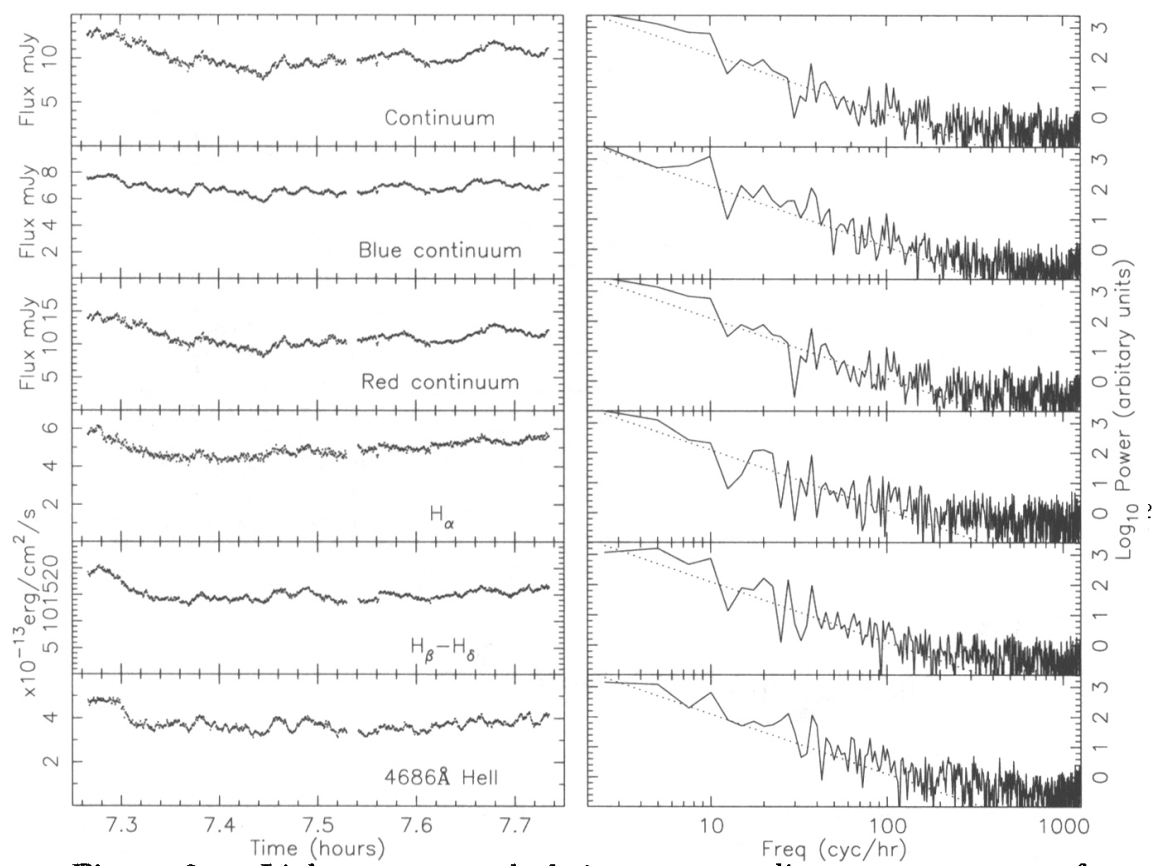

Figure 2. Light curves and their corresponding power spectra for several wavelength ranges. The continuum was subtracted before constructing the emission line light curves. Data were binned into 2.3s before constructing light curves, so each point represents 32 spectra. The dotted line shows the slope of a power law with $P(f) \propto f^{-2}$.

trograph aperture (5.2 arc seconds square) was larger than the seeing disc, and any movements of the target within the aperture due to seeing or telescope tracking/guiding adjustments caused a corresponding movement of the image on the detector. Movements along the dispersion direction reveal themselves in small wavelength displacements or 'wavelength jittering' of the spectrum. Movements along the readout direction reveal themselves in small variations of the integrated flux. The image size in the readout direction was larger than the pixel scale of 0.215 arc sec per pixel, causing some smoothing in the time direction.

'Wavelength jittering' was examined by cross correlating the mean telluric line spectrum with each individual spectrum to measure the shift. The individual spectra appeared to wander or drift randomly on timescales of approximately 1 second and with amplitudes around 0.2 pixels. A correction was applied to each spectrum accordingly. Figure 1 shows the measured shifts. No periodic signals were found in the wavelength jittering.

Small oscillations with periods between 1.1s and 1.3s were found in light curves of several different targets observed using the fast drift mode. The small oscillations were identified as instrumental effects. The AM Her data has a very small oscillation at 1.16s. The data were time binned where appropriate in order to remove these effects. The wavelength jittering and rapid oscillations and their respective correction and treatment are described in Skidmore et al. (2003). 


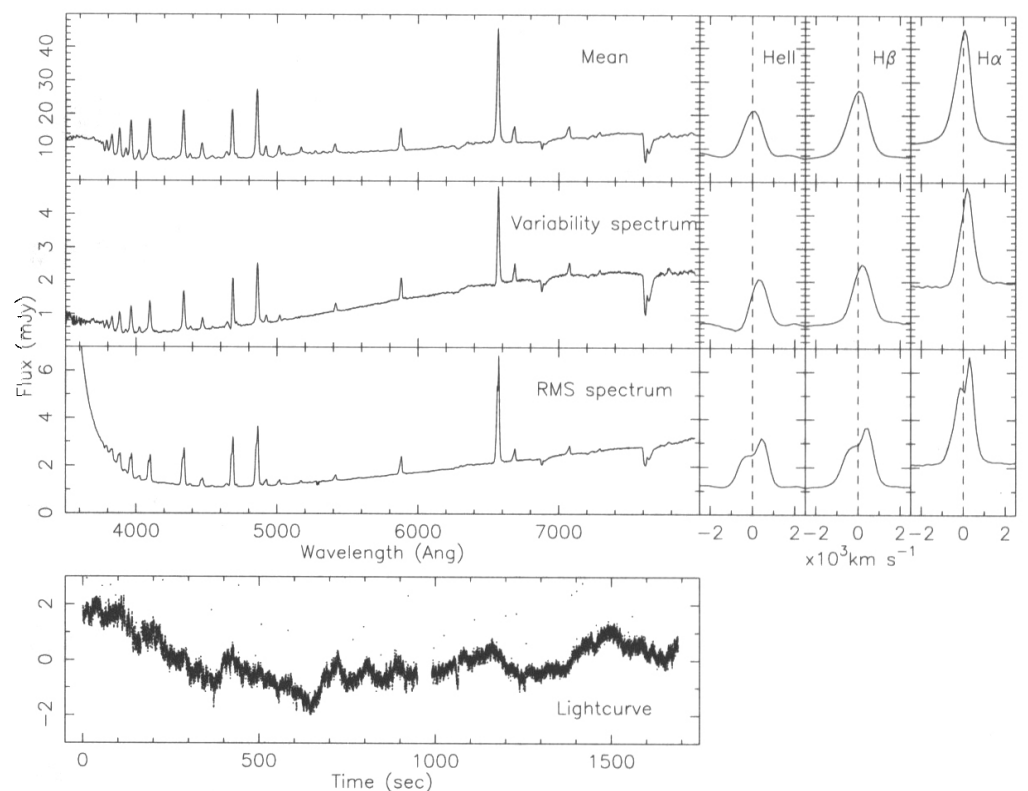

Figure 3. The mean, variability and RMS spectrum of AM Her with higher resolution plots of the emission lines and the corresponding variability light curve. The resolution is about $9 \AA$ or $500 \mathrm{~km} \mathrm{~s}^{-1}$.

The PSF was found to be a function of wavelength. The PSF toward the blue is fairly Gaussian with a FWHM of about $9 \AA$. Toward the red the PSF becomes skewed resulting in a leakage of flux toward the red. We quantified this by analysing the arc calibration spectra.

\section{Observations of AM Her \& Results}

\subsection{Light curves}

AM Her was observed on 1998 July 2 for 1689 s covering orbital phases 0.388 to 0.539 (calculated from the ephemeris of Schwarz et al. (2002)). Figure 2 shows light curves covering different wavelengths and their corresponding power spectra. All light curves have the same basic features. The blue continuum and line light curves are very similar in appearance with slight differences in the relative sizes of rapid variations in each light curve. The red continuum light curve has small short-timescale differences compared to the other light curves, i.e. at UT $7.28,7.46 \& 7.68$. The power spectra of the light curves all have very similar power law slopes. The rapid variability is probably due to the discrete but random flow of blobs onto the pole.

\subsection{Variability Spectra}

We quantify the spectral variability of AM Her using both the RMS spectrum and by decomposing the data into a constant component and a variable component such that 


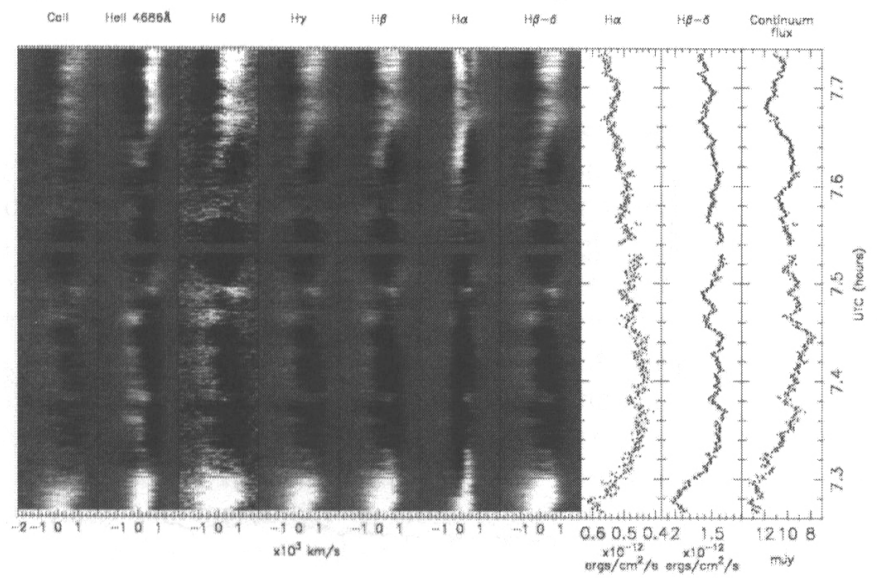

Figure 4. Trailed spectra showing line wing behaviour and light curves showing emitted flux. White denotes greater than average flux, black denotes less than average flux.

$$
F(\lambda, t)=F_{0}(\lambda)+F_{v}(\lambda) \times L(t)
$$

Figure 3 shows the mean spectrum of AM Her (as measured during our observations) and the corresponding variability spectrum $F_{v}(\lambda)$, the RMS spectrum and variability light curve, $\mathrm{L}(\mathrm{t})$. The mean spectrum has strong Balmer lines and the Balmer jump is in emission. We also see strong HeII and weaker HeI emission, typical of polars. The RMS spectrum rises sharply in the blue due to the effect of noise increasing the RMS where detector efficiency is low. The emission line variability is 'red shifted' with respect to the mean spectrum.

The emission line equivalent width is lower in the variability spectrum than in the mean spectrum, indicating that the variable component is optically thick compared to main line emitting regions. The emission line widths in the variability spectrum are narrower than in the mean spectrum, whereas in the RMS spectrum the emission line widths are greater. The residuals remaining after applying the above decomposition rise around the emission lines and are broader than the RMS spectrum emission line profiles, indicating that the decomposition fails to adequately describe the line wing variability. Thus the line wings vary on a significantly different timescale and manner to the continuum, this difference becoming larger toward the blue. The rise in the variability spectrum of AM Her to the red is most probably due to the rapid variability of the cyclotron flux.

\subsection{Trailed spectra}

Trailed spectra are shown in Figure 4. The mean spectrum has been removed and the remaining continuum has also been subtracted in order to highlight the 


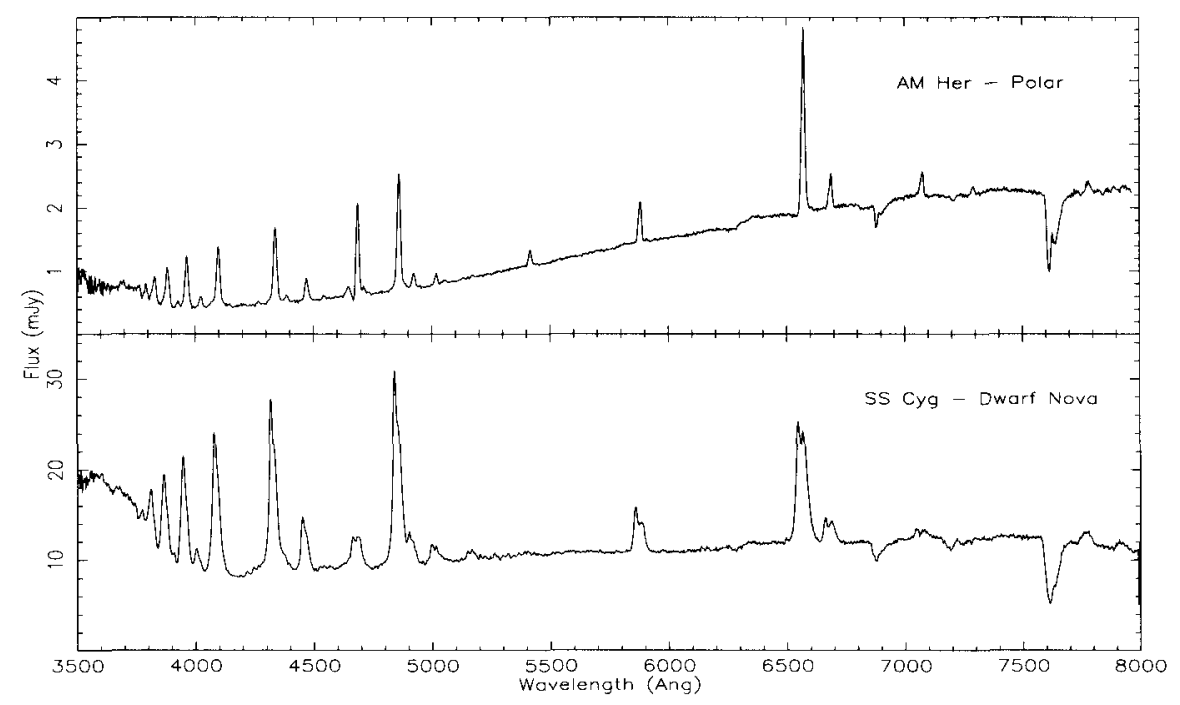

Figure 5. The variability spectrum of AM Her compared to that of SS Cyg.

velocity signatures of variations in the emission lines. Increases in line flux at the beginning and end of the run are obvious. The velocity behaviour during these brightenings suggests a blue to red transition across the line profile. However, the $\mathrm{H} \alpha$ line emission behaviour is different from that of the other lines. These differences are not due to the variable PSF mentioned above (and in fact are in a completely opposite sense to any expected effects).

Many features in the light curves coincide with features in the trailed spectra. Flares and flickers in the light curves have counterparts in the trailed spectra. However, separate features can be either red or blue shifted, e.g. the small flares at 7.47 and 7.49. At the end of the run (phase 0.539), a feature appears in the higher Balmer lines, CaII and HeII with a velocity of $700 \mathrm{~km} \mathrm{~s}^{-1}$ while the main part of the $\mathrm{H} \alpha$ emission remains at zero velocity.

During the orbital phases covered in this run $(0.388-0.539)$, any line emission from the secondary star will be red shifted as the secondary moves away, and the emission will move to zero velocity as the secondary's motion becomes transverse to the line of sight. However, this change in velocity will only be of the order of $100 \mathrm{~km} \mathrm{~s}^{-1}$ as $\mathrm{K}_{2} \simeq 200 \mathrm{~km} \mathrm{~s}^{-1}$.

Blobs moving down the ballistic stream at the observed orbital phases should be seen as a red feature moving to the blue. We do not see any features like this. As blobs enter the magnetosphere and change in velocity to follow the field lines, their line emission should move from blue to red. A long lived component can be seen moving from blue to red between 7.61 and 7.68. Further rapid spectroscopy at different orbital phases is needed to test this idea. 


\section{Discussion}

The interpretation of the trailed spectra is not particularly simple. Several possible mechanisms have been suggested during informal conversations, including variability at the threading region, coronal mass ejections, reprocessing of X-ray flares from the stream, blobs changing direction relative to the observer as they move down the stream, partial obscuration of emitting regions by separate mini streams and two blobs going in different directions around the field (two pole accretion). Detailed modeling including the prediction of the observed trailed spectrum for each mechanism is needed.

Figure 5 shows the variability spectrum of AM Her and SS Cyg, a dwarf nova during quiescence. The different size of the Balmer jump in the variability spectrum from each system is very marked, as are the relative strengths, the shapes of the Balmer lines and the very red continuum in AM Her. In AM Her the strength of the variability in HeII $4686 \AA$ is larger than that in SS Cyg and AM Her also exhibits variability in HeII 5411 $\AA$. The variability in SS Cyg is thought to arise primarily due to flickering within the accretion disc. Thus the differences in the two variability spectra shown in Figure 4 illustrate the very different processes causing the variability in AM Her and SS Cyg.

\section{Conclusions}

We have shown rapid line flux and profile changes in AM Her revealing that individual flares and flickers arise in kinematically distinct regions resulting in velocity profiles that are red or blue shifted with respect to the mean velocity.

Therefore either individual X-ray flares have distinct angular patterns of irradiation, flashing different subsets of the gas stream, or the gas stream geometry changes abruptly during flares to expose material with different velocities to the X-rays.

Detailed investigation of the Doppler signature of the flares over a range of binary phases may clarify the issue, enabling us to track the velocities of individual blobs of gas. Observations of unexpected spectral features shows that much remains to be understood about the behaviour of gas in the intense magnetic fields and high temperatures found in magnetic cataclysmic variables.

\section{References}

Beardmore A. \& Osborne J., 1997, MNRAS, 290, 145

Schwarz R., Hedelt P., Rau A., Staude A. \& Schwope A., 2002, ASP Conf. Proc., 261,167

Schwope A., 2001, Lecture Notes in Physics, pub. Springer, 573, 127

Skidmore W., O'Brien K., Horne K., Gomer R., Oke J. \& Pearson K., 2003, MNRAS, 338, 1057. 